\title{
Outcome and toxicity of radical radiotherapy or concurrent Chemoradiotherapy for elderly cervical cancer women
}

Weiping Wang, Xiaorong Hou, Junfang Yan, Jie Shen, Xin Lian, Shuai Sun, Zhikai Liu, Qingyu Meng, Dunhuang Wang, Mei Zhao, Jie Qiu, Ke Hu* and Fuquan Zhang*

\begin{abstract}
Background: Concurrent chemoradiotherapy (CCRT) is the standard treatment for local advanced cervical cancer. However, for elderly patients, studies are limited and the outcomes are controversial. We retrospectively analyzed the efficacy and tolerance of radical radiotherapy (RT) or CCRT in elderly cervical cancer patients and performed comparisons between them.

Methods: We retrospectively analyzed the elderly cervical cancer patients ( $\geq 70$ years old) treated with radical RT or CCRT between January 2006 and December 2014. For external beam radiotherapy, 50Gy in 25 fractions or 50.4Gy in 28 fractions were delivered via 3-dimensional conformal radiation therapy or intensity modulated radiation therapy. High-dose-rate intracavitary brachytherapy was performed with a dose of 30-36Gy in 5-7 fractions to point A. Concurrent chemotherapy regimens included weekly cisplatin and paclitaxel.

Results: Seventy-three patients were eligible for this study. Twenty-one(28.8\%) and 52(71.2\%) patients suffered with FIGO stage IB-IIA and IIB-IVA disease, respectively. Twenty-four (32.9\%) patients received CCRT. The median duration of follow-up was 32.4 months (4.8-118.8 months). The 3-year overall survival (OS), cancer-specific survival (CSS) and disease-free survival (DFS) were $64.9 \%, 67.8 \%$ and $66.5 \%$, respectively. By multivariate analysis, CCRT was a significant predictive factor of OS( $p=0.023,95 \%$ confidence interval [Cl]: 1.172-8.860), $\operatorname{CSS}(p=0.031,95 \% \mathrm{Cl}$ : 1.131-13.908)and $\operatorname{DFS}(p=0.045,95 \% \mathrm{Cl}: 1.023 \sim 6.430)$. The 3 -year OS of patients received RT and CCRT were $54.3 \%$ and $83.1 \%$, CSS were $56.8 \%$ and $87.1 \%$, DFS were $57.6 \%$ and $83.3 \%$. There was no treatment related death. Grade 3-4 acute hematological, gastrointestinal and urinary toxicity incidences were $31.5 \%, 19.1 \%$ and $12.3 \%$, respectively. For grade 3-4 chronic gastrointestinal and genitourinary toxicities, the incidences were $4.1 \%$ and $2.7 \%$, respectively. Compared with RT, CCRT was related with high grade $3-4$ hematological toxicity ( $16.3 \%$ and $62.5 \%$ respectively, $p<0.001$ ), respectively. However, acute nonhematological toxicity and chronic toxicity were not significantly different.

Conclusion: Elderly cervical cancer patients could tolerate radical RT and CCRT very well and get a favored survival. Compared with RT, CCRT could improve the survival of elder cervical cancer patients with similar nonhematological toxicity. CCRT should be considered in elderly cervical cancer patients.
\end{abstract}

Keywords: Elderly cervical cancer, Radical radiotherapy, Chemoradiotherapy

\footnotetext{
* Correspondence:

huk@pumch.cn; zhangfuquan3@sina.com; zhangfuquan3@126.com

Department of radiation oncology, Peking Union Medical College Hospital.

Chinese Academy of Medical Sciences \& Peking Union Medical College, NO.1

Shuaifuyuan Wangfujing, Dongcheng District, Beijing 100730, People's

Republic of China
} 


\section{Background}

In China, cervical cancer was the seventh most common cancer in women, with an estimated 98.9 thousands new cases and 30.5 thousands deaths in 2015 [1]. It is usually considered that cervical cancer is uncommon in elderly women. The incidence is similar from the third decade to 85 years old. However, the data from China showed that women older than 60 years old accounted for $23.8 \%$ of all the cervical cancer patients [1], probably associated with the aged tendency of population. Despite the dramatical decrease of incidence of cervical cancer in US during the recent years, incidence in elderly women has no significant reduction [2]. A more tough situation concerning is that the incidence of cervical cancer was still increasing in China [1].

Compared with young patients, elder patients are more likely to have more advanced disease, and receive less aggressive treatment. A research based on Surveillance, Epidemiology, and End Results (SEER) database showed that, for patients at the age of $<50$, $50-59,60-69,70-79$ and $\geq 80$ years old, the proportions of the International Federation of Gynecology and Obstetrics (FIGO) stage I patients were 70.1\%, $49.2 \%, 45.7 \%, 39.9 \%$ and $33.2 \%$, respectively, while proportions of patients with FIGO stage IIIB disease were $6.7 \%, 13.8 \%, 13.3 \%, 14.9 \%$ and $16.9 \%$, respectively. Elderly patients were more frequently diagnosed with advanced diseased. For Stage IIB-IVA women, no treatment was allocated to $3.9 \%$ of patients $<50$ years old, compared with $7.3 \%$ of women aged $70-79$ and $12.1 \%$ of the women $\geq 80$ years old $(p<0.0001)$. The use of brachytherapy also declined with age $(p<0.0001$, [3]. A research from Brazil also demonstrated that elderly patients were less likely to receive surgery, chemotherapy, brachytherapy, and more likely to receive no treatment [4].

For local advanced cervical cancer (stage IB2, IIA2, IIB-IVA), cisplatin-based concurrent chemoradiotherapy(CCRT) is the standard treatment at present [5]. However, elderly patients always suffer from weakness, malnutrition, comorbidity and so on, which may increase the incidence of treatment complications. Some elderly patients are unable to tolerate surgery or refuse surgery because of concerns about complications. Most elderly patients have no desire of reproduction or ovaries conservation. So some early stage patients may choose radical radiotherapy (RT) or CCRT as primary treatment other than surgery. Therefore, elderly patients have more demands for radical RT or CCRT. On the outcome and toxicity of CCRT for elderly patients, studies are limited at present [6-8].

In the present study, we retrospectively analyzed the efficacy and tolerance of elderly cervical cancer patients treated with radical RT or CCRT. We also performed comparisons between RT and CCRT.

\section{Methods}

Cervical cancer patients treated with radical RT or CCRT in Peking Union Medical College Hospital (PUMCH) between January 2006 and December 2014 were retrospectively reviewed. The inclusion criteria were as follows: cervical cancer diagnosed by histopathology of biopsy; no evidence of distant metastasis; no previous surgery or radiotherapy for cervical cancer; $\geq 70$ years old. Patients who underwent only palliative RT were excluded from this study.

\section{Radiotherapy}

Patients underwent CT (16-slice Philips Brilliance Big Bore CT) simulation with intravenous and oral contrast agents; Rectum, bladder preparation and virginal marker were performed before CT simulation. The clinical target volume (CTV) and gross tumor volume (GTVnd) were contoured on the individual axial CT slices for each patient. The GTVnd included pelvic/para-aortic metastatic lymph nodes. The criteria for metastatic lymph nodes were: short diameter longer than $1 \mathrm{~cm}$; proved by functional imaging technique like positron emission tomography or diffusion-weighted magnetic resonance imaging (MRI). The CTV covered the gross tumor, cervix, uterus, parametrium, upper part of the vagina to $3 \mathrm{~cm}$ below the tumor invasion, and regional lymph nodes (common iliac, internal iliac, external iliac, obturator, presacral, and/or para-aortic lymph nodes). A 5$\mathrm{mm}$ margin was added to the GTV to create the final planning gross tumor volume (PGTVnd). The planning target volume (PTV) was defined as the CTV plus an 8-10 $\mathrm{mm}$ margin and an additional 5-10 $\mathrm{mm}$ margin to the cervix and uterus.

Three-dimensional conformal radiation therapy (3DCRT) was delivered using 15- or 18-MV photons with a four-field box technique. A total dose of 50Gy (2Gy per fraction) was prescribed to the PTV. A 4-cm central shield was used after 36-40Gy to shield the rectum and bladder. Intensity modulated radiation therapy (IMRT) was delivered using 6-MV photons. A dose of 50.4Gy in 28 fractions was prescribed to the PTV. A concomitant boost to 59-61Gy was delivered to the PGTVnd for patients receiving IMRT. At least $95 \%$ of the final PTV or PGTV received $100 \%$ of the prescribed dose and at least $100 \%$ of the CTV or GTV was to receive $100 \%$ of the dose. For patients with IMRT, a second CT simulation and treatment planning were performed after 20 fractions external beam radiation therapy (EBRT).

The source used in intracavitary brachytherapy (ICBT) was iridium-192. ICBT generally began after 3 weeks of EBRT, 1-2 fractions per week. A cumulative dose of 3036Gy/5-7 fractions was prescribed to point A according to International Commission of Radiation Units (ICRU) 38. Patients received conventional orthogonal film and 
brachytherapy planning after every insertion. CT was performed to check the applicator position after the first insertion.

\section{Chemotherapy}

For patients receiving concurrent chemotherapy, the first line regimen was cisplatin $\left(30-40 \mathrm{mg} / \mathrm{m}^{2} /\right.$ week). For patients with renal dysfunction, paclitaxel (60$80 \mathrm{mg} / \mathrm{m}^{2} /$ week) was administered.

\section{Follow-up and evaluation of toxicity}

Patients underwent gynecological examination and pelvic MRI/CT one month after treatment. After that, patients had follow-up examinations approximately every 3 months for the next 2 years, every 6 months for 3-5 years after treatment, and then once a year. The acute and chronic toxicity were evaluated with Common Terminology Criteria for Adverse Events (CTCAE) version 3.0.

\section{Statistical analysis}

Survival rate was measured from the completion of treatment. Overall survival (OS), cause-specific survival (CSS) and disease-free survival (DFS) were estimated using the Kaplan-Meier method. The significance of difference was examined with a log-rank test. Cox's proportional hazard model was used for the multivariate analysis. We used chi-square test, continuity correction and the Fisher exact test to compare the toxicity of radical RT and CCRT. Differences were considered statistically significant at $p<0.05$. Statistical analysis was performed using SPSS v.19.0.

\section{Results}

\section{Patient characteristics and treatment}

During the time period from January 2006 and December 2014, there were 73 patients eligible for the research. The median age of patients was 74 years old, ranging from 70 to 88 years old. The majority of patients (68 patients, 93.2\%) had squamous cell carcinoma, 4 patients (5.5\%) with adenocarcinoma and 1 patient $(1.4 \%)$ with small cell carcinoma. The FIGO stage ranged from IB to IVB. Nine (12.3\%), 12 (16.4\%) and 52 (71.2\%) patients suffered with FIGO stage IB, IIA and IIB-IVA disease, respectively. There were 11 patients (15.1\%) with positive lymph nodes, 4 (5.5\%) of them with positive para-aortic lymph nodes. The detailed patients and tumor characteristics are shown in Table 1.

3D-CRT and IMRT were performed to $21(28.8 \%)$ and 52 patients $(71.2 \%)$ respectively. The ICBT dose was 30 Gy or higher in 54 patients $(74.0 \%)$. The radiotherapy wasn't completed in 3 patients. One of them didn't complete radiotherapy because of grade 3 gastrointestinal(GI) toxicity, and this patient received extended-field
Table 1 Patients, tumor and treatment characteristics

\begin{tabular}{|c|c|c|c|}
\hline & Characteristic & $\begin{array}{l}\text { No. of } \\
\text { patients }\end{array}$ & $\begin{array}{l}\text { Percentage } \\
(\%)\end{array}$ \\
\hline \multirow[t]{2}{*}{ Age (years old) } & Median & 74 & \\
\hline & $\begin{array}{l}70-74 \\
75-79 \\
\geq 80\end{array}$ & $\begin{array}{l}44 \\
20 \\
9\end{array}$ & $\begin{array}{l}60.3 \\
27.4 \\
12.3\end{array}$ \\
\hline \multirow[t]{3}{*}{ Histology } & $\begin{array}{l}\text { Squamous cell } \\
\text { carcinoma }\end{array}$ & 68 & 93.2 \\
\hline & Adenocarcinoma & 4 & 5.5 \\
\hline & $\begin{array}{l}\text { Small cell } \\
\text { carcinoma }\end{array}$ & 1 & 1.4 \\
\hline \multirow[t]{6}{*}{ Stage } & $\mathrm{IB}$ & 9 & 12.3 \\
\hline & $\| A$ & 12 & 16.4 \\
\hline & $\| \mathrm{B}$ & 32 & 43.8 \\
\hline & $\| \mathrm{A}$ & 6 & 8.2 \\
\hline & $\| \mathrm{B}$ & 9 & 12.3 \\
\hline & IVA & 5 & 6.8 \\
\hline \multirow[t]{3}{*}{ LNM } & Pelvic LNM & 7 & 9.6 \\
\hline & Para-aortic LNM & 2 & 2.7 \\
\hline & $\begin{array}{l}\text { Pelvic and } \\
\text { para-aortic LNM }\end{array}$ & 2 & 2.7 \\
\hline \multirow{2}{*}{$\begin{array}{l}\text { External beam radiation } \\
\text { therapy technique }\end{array}$} & 3D-CRT & 21 & 28.8 \\
\hline & IMRT & 52 & 71.2 \\
\hline \multirow{3}{*}{$\begin{array}{l}\text { Dose of intracavitary } \\
\text { brachytherapy }\end{array}$} & $<30$ Gy & 19 & 26.0 \\
\hline & 30-36 Gy & 50 & 68.5 \\
\hline & $>36$ Gy & 4 & 5.5 \\
\hline \multirow[t]{2}{*}{ Completion of radiotherapy } & Yes & 70 & 95.9 \\
\hline & No & 3 & 4.1 \\
\hline \multirow[t]{2}{*}{ Duration of radiotherapy } & $\leq 8$ weeks & 58 & 79.5 \\
\hline & $>8$ weeks & 15 & 20.5 \\
\hline \multirow[t]{3}{*}{ Concurrent chemotherapy } & No & 49 & 67.1 \\
\hline & $1-3$ cycles & 13 & 17.8 \\
\hline & $\geq 4$ cycles & 11 & 15.1 \\
\hline
\end{tabular}

Abbreviations: LNM Lymph nodes metastasis, 3D-CRT 3-Dimensional conformal radiation therapy, IMRT Intensity modulated radiation therapy

RT and concurrent chemotherapy of cisplatin regimen. The other 2 patients treated with radical RT without concurrent chemotherapy refused to continue ICBT. One of them underwent radical surgery after 36Gy EBRT in 20 fractions and 6Gy ICBT in 1 fraction. The overall duration of radiotherapy ranged from 26 to 87 days with a median of 50 days. For patients received RT and CCRT, the median duration was 49 and 50 days respectively. The duration was more than 8 weeks for 15 patients $(20.5 \%)$, including 11 patients $(22.4 \%)$ treated with RT and 4 patients (16.7\%) treated with CCRT.

Twenty-four patients (32.9\%) received concurrent chemotherapy. Cisplatin was administered for 21 patients and paclitaxel for the other 3 patients. Eleven 
patients (15.1\%) underwent $\geq 4$ cycles weekly chemotherapy and the other 13 (17.8\%) with 1-3 cycles. For these 13 patients, the reason for less than 4 cycles chemotherapy included acute hematological toxicity lasting several weeks ( 4 patients), grade 3 or higher GI toxicity (4 patients), renal failure (1 patients), refusing to continue chemotherapy without severe acute toxicity (4 patients). The treatment characteristics are detailed in Table 1.

\section{Outcome and pattern of failure}

The median duration of follow-up was 32.4 months (4.8-118.8 months). The 3-year OS, CSS and DFS were $64.9 \%, 67.8 \%$ and $66.5 \%$, respectively.

At the end of follow-up, 24 patients (32.8\%) experienced tumor relapse. Eleven patients (15.1\%) had locoregional failure, 9 patients (12.3\%) experienced distant metastasis, and 4 (5.5\%) had locoregional failure and distant metastasis. Of the 15 patients (20.5\%) with locoregional failure, 6 patients didn't achieve completely response (CR) after treatment. Nine patients experienced locoregional relapse after CR. For patients with locoregional failure, 2 patients had stage IIA disease, 5 patients with stage IIB disease, 1 patient with stage IIIA disease, 5 with stage IIIB disease and 2 patients with stage IVA disease. Among the 13 patients (17.8\%) with distant metastasis, distant metastasis sites included lung (5 patients), para-aortic lymph nodes (3 patients), mediastinum lymph nodes (3 patients), liver (1 patient) and bone (1 patient).

Five $(20.8 \%)$ of the 24 patients with tumor relapses $(4$ patients treated with CCRT and 20 patients treated with $\mathrm{RT}$ as initial treatment) received systemic chemotherapy for their relapses. One of the 4 patients $(25 \%)$ treated with CCRT before received chemotherapy. As for the 20 patients treated with RT before, 4 patients $(20 \%)$ underwent chemotherapy. The regimens included ciplatin, cisplatin and paclitaxel, cisplatin and fluorouracil.

At the time of last follow-up, 27 patients (36.9\%) died. Twenty patients $(27.4 \%)$ of them died of cervical cancer and 7 patients (9.6\%) died of other causes, including heart failure ( 2 patients), meatus urinarius melanoma (1 patient), pulmonary embolism (1 patient), chronic obstructive pulmonary diseases (1 patient), cerebral hemorrhage (1 patient) and accidental death (1 patient).

\section{Prognostic factors}

The results of univariate analysis are summarized in Table 2. The results indicated that FIGO stage, regional lymph nodes metastasis and CCRT were significant factors of OS, CSS and DFS. The 3-year OS of radical RT and CCRT were $54.3 \%$ and $83.1 \%(p=0.0038)$, 3year CSS were $56.8 \%$ and $87.1 \%(p=0.0061), 3$-year DFS were $57.6 \%$ and $83.3 \%(p=0.0091)$, respectively. The
OS, CSS and DFS curves of patients treated with RT and CCRT are shown in Fig. 1. The results of multivariate analysis are presented in Table 3. FIGO stage was an independent factor of DFS $(p=0.005$, 95\%confidence interval[CI]: 1.232-3.188). Regional lymph nodes metastasis were associated with worse OS $(p=0.001,95 \% \mathrm{CI}$ : 1.786-11.556), CSS ( $p<0.001,95 \%$ CI: $2.156 \sim 15.443)$ and DFS ( $p=0.001,95 \%$ CI: $1.817-11.017)$, CCRT was a significant predictive factor of OS $(p=0.023,95 \% \mathrm{CI}$ : $1.172-8.860)$, CSS $(p=0.031,95 \%$ CI: $1.131-13.908)$ and DFS ( $p=0.045,95 \%$ CI: $1.023 \sim 6.430$ ).

\section{Toxicities}

As shown in Tables 4, 25 patients (31.5\%) experienced Grade 3 or higher acute hematological toxicity, the incidences in patients treated with RT and CCRT were 16.3\% and $62.5 \%$, respectively $(p<0.001)$. For 24 patients treated with CCRT, incidences of grade 3 or greater hematological toxicity of patients treated with 3D-CRT (7 patients) and IMRT (17 patients) were $71.4 \%$ and $58.8 \%(p=0.908)$. There were 14 patients (19.1\%) developed acute grade 3 or greater GI toxicity. The incidences were similar between RT (18.4\%) and CCRT (20.8\%). Nine patients $(12.3 \%)$ experienced grade 3 or greater acute urinary toxicity. The incidence of patients treated with CCRT (16.7\%) was higher than RT (10.2\%). However, the difference wasn't significant $(p=0.705)$.

Four patients (5.5\%) developed grade 3-4 chronic toxicity, including 1 patient with vesicorectal fistula, 1 patient with vaginorectal fistula, and 2 patients with proctitis. The patient with vesicorectal fistula suffered with stage IIB cervical cancer and was treated with 50.4Gy IMRT to the pelvic combine with 36Gy in 6 fractions ICBT. This patient developed vesicorectal fistula 15 months after treatment without cancer recurrence and received cystostomy and partial rectectomy. Another patient with stage IIIA disease was treated 50Gy in 25 fractions pelvic irradiation and 32.5Gy in 6 fractions ICBT combined with concurrent chemotherapy. The patient acquired CR after treatment and suffered with vaginal relapse 37.6 months after treatment. Then 30Gy in 15 fractions IMRT and 15Gy in 3 fractions ICBT were prescribed to the vaginal. The patient developed vaginorectal fistula 7 months after the second irradiation. She received surgery repair and had another 61.3 months disease-free survival.

The incidences of grade 3 or greater GI and genitourinary (GU) toxicity were $4.1 \%$ and $2.7 \%$, respectively. The chronic GI and GU toxicity of CCRT was not significantly higher than RT.

\section{Discussion}

It is controversial whether the survival of elderly cervical cancer patients is poorer than young patients. 
Table 2 Unvariate analysis of factors influencing OS, CSS and DFS

\begin{tabular}{|c|c|c|c|c|c|c|c|}
\hline Factors & No. & 3-year OS (\%) & $p$ & 3-year CSS (\%) & $p$ & 3-year DFS (\%) & $p$ \\
\hline \multicolumn{8}{|l|}{ Age (years old) } \\
\hline $70-74$ & 44 & 64.0 & 0.7516 & 65.8 & 0.8542 & 66.1 & 0.9648 \\
\hline$\geq 75$ & 29 & 65.8 & & 70.0 & & 67.5 & \\
\hline \multicolumn{8}{|l|}{ Histology } \\
\hline Squamous cell carcinoma & 68 & 65.9 & 0.3010 & 68.9 & 0.2478 & 67.3 & 0.3806 \\
\hline Other histology & 5 & 50.0 & & 50.0 & & 60.0 & \\
\hline \multicolumn{8}{|l|}{ Stage } \\
\hline । & 9 & 88.9 & 0.0004 & 88.9 & 0.0074 & 88.9 & $<0.0001$ \\
\hline$\|$ & 44 & 72.6 & & 72.6 & & 74.8 & \\
\hline III & 15 & 55.1 & & 60.1 & & 53.3 & \\
\hline IV A & 5 & 20.0 & & 0 & & 0 & \\
\hline \multicolumn{8}{|l|}{ Lymph nodes metastasis } \\
\hline Yes & 11 & 27.3 & $<0.0001$ & 0 & $<0.0001$ & 18.2 & $<0.0001$ \\
\hline No & 62 & 75.1 & & 78.7 & & 75.5 & \\
\hline \multicolumn{8}{|c|}{ External beam irradiation technique } \\
\hline 3D-CRT & 21 & 57.1 & 0.4969 & 61.2 & 0.5433 & 57.1 & 0.2360 \\
\hline IMRT & 52 & 70.5 & & 72.2 & & 71.6 & \\
\hline \multicolumn{8}{|l|}{ Concurrent chemotherapy } \\
\hline Yes & 24 & 83.1 & 0.0038 & 87.1 & 0.0061 & 83.3 & 0.0091 \\
\hline No & 49 & 54.3 & & 56.8 & & 57.6 & \\
\hline
\end{tabular}

Abbreviations: OS Overall survival, CSS Cause-specific survival, DFS Disease-free survival, LNM Lymph nodes metastasis, 3D-CRT 3-Dimensional conformal radiation therapy, IMRT Intensity modulated radiation therapy

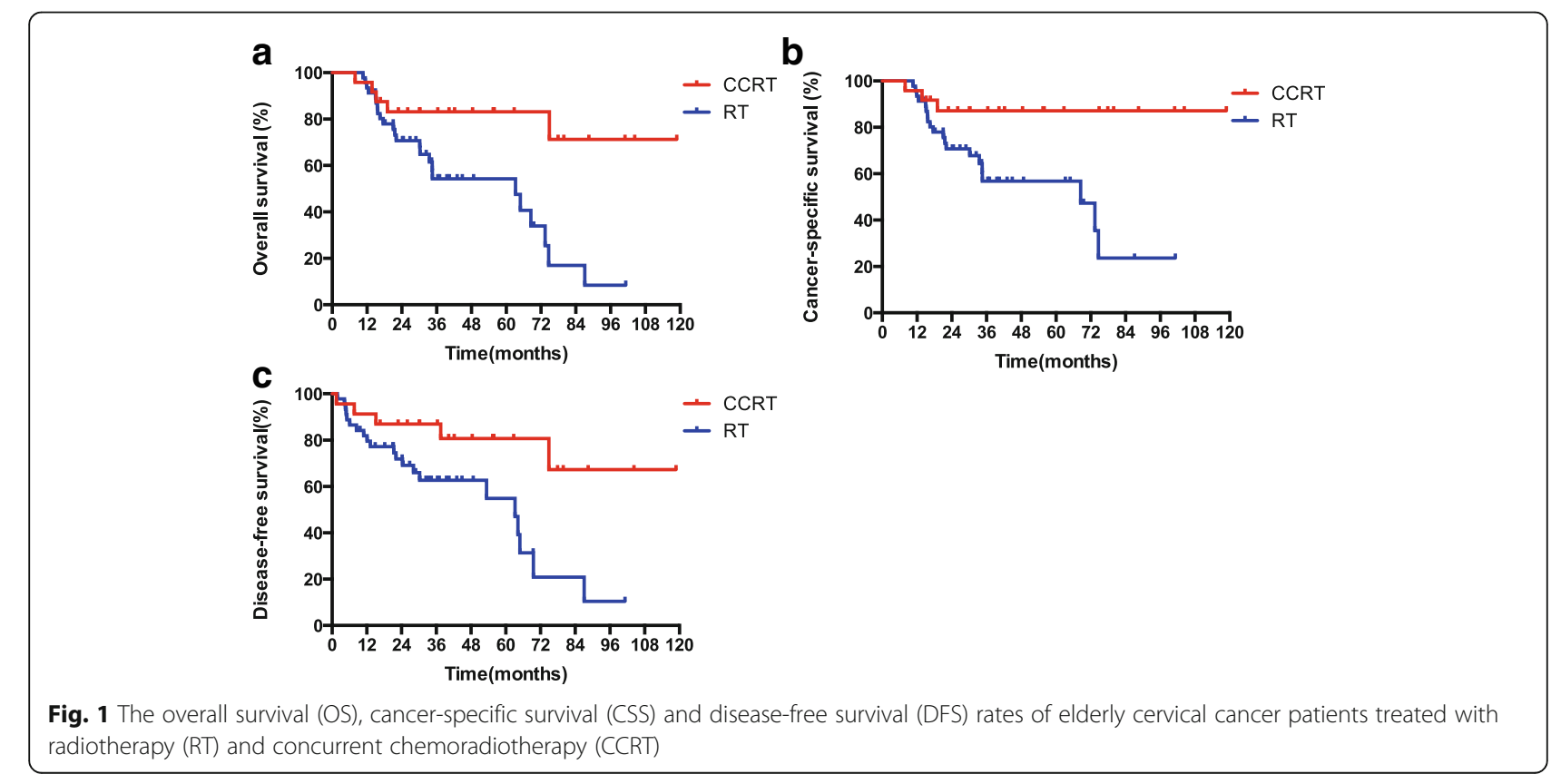


Table 3 Multivariate analysis of factors influencing OS, CSS and DFS

\begin{tabular}{|c|c|c|c|c|c|c|}
\hline \multirow[t]{2}{*}{ Factors } & \multicolumn{2}{|l|}{ OS } & \multicolumn{2}{|l|}{ CSS } & \multicolumn{2}{|l|}{ DFS } \\
\hline & HR $(95 \% \mathrm{Cl})$ & $p$ & $\mathrm{HR}(95 \% \mathrm{Cl})$ & $p$ & $\mathrm{HR}(95 \% \mathrm{Cl})$ & $p$ \\
\hline Stage $(I \sim \mathrm{IV})$ & $1.539(0.945 \sim 2.508)$ & 0.083 & $1.370(0.795 \sim 2.362)$ & 0.257 & $1.982(1.232 \sim 3.188)$ & 0.005 \\
\hline Lymph nodes metastasis (Yes VS No) & $4.543(1.786 \sim 11.556)$ & 0.001 & $5.770(2.156 \sim 15.443)$ & $<0.001$ & $4.474(1.817 \sim 11.017)$ & 0.001 \\
\hline Concurrent chemotherapy(Yes VS No) & $3.223(1.172 \sim 8.860)$ & 0.023 & $3.967(1.131 \sim 13.908)$ & 0.031 & $2.565(1.023 \sim 6.430)$ & 0.045 \\
\hline
\end{tabular}

Abbreviations: OS Overall survival, CSS Cause-specific survival, DFS Disease-free survival, HR Hazard ratio, CI Confidence interval

In retrospectively study with large population, the survival of elderly patients was always poorer than younger patients [3, 4, 9]. Maybe it was because elderly patients suffered from more advanced disease and treated with less aggressive treatment [3, 4, 9]. When treated similarly (such as RT or CCRT), the survival of young and elderly patients were similar [6, 10-12]. In this study, we compared the outcome of patients aged $70-74$ and $\geq 75$, the OS, CSS and DFS were not significantly different. This may indicate that, elderly patients can get a good survival if they received adequate treatment.

RT has been considered as an important primary treatment approach of cervical cancer for more than half a century. We have many experiences on the efficacy and tolerance of RT for elderly patients. It was reported that elderly patients could get equivalent survival to young patients when treated with RT [10-12]. A propensity score-matched study showed no significant differences in CSS, local failure and distant failure rates between elderly group ( $\geq 75$ years) and young group ( $<60$ years), although OS was worse in the elderly patients. The 5year CSS of elderly and young groups were $73.1 \%$ and $76.2 \%$, respectively $(p=0.456)$ [12]. Some studies reported that treatment toxicity was not significantly related with age $[10,11]$ while some others showed that age was a significant factor for radiation complications [12]. Ikushima, $\mathrm{H}$, et al. repoted that the incidences of grade 2 or greater chronic toxicity was $22 \%, 31 \%$ and $8 \%$ respectively for patients aged $\leq 64 、 65-74$ and $\geq 75$ years old [10]. In the study of Wang YM et al., elderly patients experienced more grade 3 procitis compared with young patients $(18.1 \%$ and $6.2 \%$, respectively; $p=0.040$ [12]. In our study, the incidences of grade 3 or greater acute hematological, GI and urinary toxicities were $16.3 \%$,
$18.4 \%$ and $10.2 \%$ respectively for patients treated with RT, which were comparatively low. Among 49 patients treated with RT, only 2 patients didn't complete the treatment because of refusal to ICBT [10-12].

From 1999, several large randomized clinical trials proved that, compared with RT, the addition of cisplatin-based concurrent chemotherapy could improve the survival of cervical cancer [13]. These studies changed the treatment approach of cervical cancer. However, only very few of elderly cervical cancer patients were included in these clinical trials [13]. Elderly patients always can't tolerate CCRT as good as young patients because of weakness, malnutrition, comorbidity and so on. However, because of the lacking clinical trials and guidelines, elderly patients were generally treated with the approach for young patients. The studies of CCRT were limited and controversial at present. Goodheart, $\mathrm{M}$ et al. compared 69 nonelderly cervical cancer patients $(<65$ years old) and 27 elderly patients ( $\geq 65$ years old) treated with RT or CCRT. It demonstrated that the toxicity were similar between the two groups and CCRT was associated with decreased mortality $(P<0.01)$. The decrease in mortality did not differ between the two age cohorts (all causes: $p=0.66$; cancer specific: $p=0.65$ ) [6]. Chakraborty $\mathrm{S}$, et al. treated 43 young cervical cancer patients ( $<65$ years) and 23 elderly patients ( $\geq 65$ years) with CCRT (98\% of young patients and $65 \%$ of elderly patients) or RT. The EBRT was IMRT for all patients. Grade 3 hematological toxicities (26.7\% versus $16.7 \%)$, GI toxicity (16.7\% versus $13.3 \%$ ), treatment breaks, treatment duration and early outcomes were not significantly different between young and elderly patients [7]. Park, J.H. et al. compared 61 elderly cervical cancer patients treated with RT and 44 elderly patients with CCRT. They found that CCRT was related with higher acute

Table 4 Acute and chronic toxicity of elderly cercal cancer patients treated with RT or CCRT

\begin{tabular}{|c|c|c|c|c|c|}
\hline & & Total & RT & CCRT & $p$ \\
\hline \multirow[t]{3}{*}{$\geq$ grade 3 acute toxicity } & Hematological toxicity & $25(31.5 \%)$ & $8(16.3 \%)$ & $15(62.5 \%)$ & $<0.001$ \\
\hline & Gastrointestinal toxicity & $14(19.1 \%)$ & $9(18.4 \%)$ & $5(20.8 \%)$ & 1.000 \\
\hline & Urinary toxicity & $9(12.3 \%)$ & $5(10.2 \%)$ & $4(16.7 \%)$ & 0.705 \\
\hline \multirow[t]{2}{*}{$\geq$ grade 3 chronic toxicity } & Gastrointestinal toxicity & $3(4.1 \%)$ & $3(4.1 \%)$ & $0(0 \%)$ & 0.546 \\
\hline & Genitourinary toxicity & $2(2.7 \%)$ & $1(2.0 \%)$ & 1 (4.2\%) & 1.000 \\
\hline
\end{tabular}


hematologic and gastrointestinal toxicity. However, the analysis showed no benefit of CCRT with respect to OS and CSS. The 5-year OS in RT group and CCRT group were $53.5 \%$ and $61.8 \%(p=0.4534)$, CSS were $66.6 \%$ and $68.8 \%(p=0.8584)[8]$.

In our study, CCRT was an independent predictive factor of OS $(p=0.023,95 \%$ CI: $1.172-8.860)$, CSS $(p=0.031,95 \%$ CI: $1.131-13.908)$ and DFS $(p=0.045$, $95 \%$ CI: $1.023 \sim 6.430)$. For patients treated with RT and CCRT, the 3-year OS were $54.3 \%$ and $83.1 \%$, 3-year CSS were $56.8 \%$ and $87.1 \%$, 3-year DFS were $57.6 \%$ and $83.3 \%$, respectively. This may indicate that CCRT could improve the survival of elderly cervical cancer patients. Since this was a retrospectively study with small population, we should interpret this result with caution.

Our study shown that CCRT was associated with more acute hematological toxicity. The acute nonhematological toxicity and chronic toxicity were similar between RT and CCRT. The incidences of grade 3 or higher hematological toxicity was $62.5 \%$ for patients treated with CCRT, which was higher than RT (16.3\%, $p<0.001)$. The hematological toxicity was also much higher than previous studies in which most patients were non-elderly cervical cancer women. This may be becasuse the tolerance to CCRT of elderly patients is not as good as young patients. It was reported that, compared with 3D-CRT, bone marrow-sparing IMRT could reduce the dose of bone marrow and hematological toxicity [14-16]. In the study of Hui B et al., in IMRT group and 3D-CRT group, the V50 of bone marrow $9.79 \%$ and $15.4 \%$, respectively. And the incidence of grade 2 or greater neutropenia was $80 \%$ and $40 \%$ in these 2 groups [14]. Considering the high hematological toxicity of CCRT, elderly patients may gain more benefits from bone marrow sparing IMRT. In our institute, we didn't spare bone marrow for cervical cancer patients treated with IMRT. Incidence of grade 3 or greater hematological toxicity of 3D-CRT (71.4\%) was higher than IMRT (58.8\%) in our study. However, it was not significant.

For patients treated with CCRT, the incidence of grade 3 or higher acute GI and urinary toxicities were $20.8 \%$ and $16.7 \%$, which were a little higher than RT $(18.4 \%$ and $10.2 \%$ ), although the difference was not significant. The grade 3 or greater chronic GI toxicity occurred in $4.1 \%$ and $0 \%(p=0.546)$ of patients treated with RT and CCRT, respectively. For grade 3 or greater GU toxicity, the incidences was $2.0 \%$ and $4.2 \%(p=1.000)$, respectively. CCRT didn't significantly increase the chronic toxicity.

Generally speaking, 4-6 cycles concurrent chemotherapy were recommended for cervical cancer women treated with CCRT. In our stury, of 24 patients treated with CCRT, 13 patients received concurrent chemotherapy less than 4 cycles. In our institute this proportion was much higher than young patients. The main reason was treatment-related toxicity (9 patients, 69.2\%). Three patients had not completed the radiotherapy, 2 of them were treated with RT and 1 with CCRT. The addition of concurrent chemotherapy didn't influence the completion of radiotherapy. The acute toxicity of CCRT may lead to interruption of radiotherapy. And longer overall treatment time may lead to poor survival [17]. National Comprehensive Cancer Network (NCCN) guideline of cervical cancer recommended that radiotheray should be completed in 8 weeks [5]. In our study, concurrent chemotherapy didn't prolong the duration of radiotherapy. The duration of radiotherapy was 49 and 50 days for patients received RT and CCRT. The duration of radiotherapy was longer than 8 weeks in $22.4 \%$ patients with RT and $16.7 \%$ patients with CCRT. Overall, most patients could tolerate CCRT very well.

During the last two decade, modern treatment approaches such as MRI based image guided brachytherapy (IGBT) and IMRT were used in the treatment of cervical cancer. Compared with 2D brachytherapy, IGBT could improve the survival with reduced severe morbidity [18-20]. A large multi-institutional study (RetroEMBRACE) involved 731 patients treated with ICBT combined with radio-chemotherapy. The study also included elderly patients. The median age was 53 years (range 23-91). IGBT combined with radiochemotherapy leaded to excellent survival and limited severe toxicity. The 3-year LC, CSS and OS were $91 \%, 79 \%$ and $74 \%$, respectively. The survival was much better than our study (3-year CSS and OS: $67.8 \%$ and $64.9 \%$ ). This study also showed that concomitant chemotherapy had a significant impact on OS [18]. Compared with anteroposterior and posteroanterior parallel portals or 4 fields "box" radiotherapy, IMRT could decrease the treatment toxicity with comparable or better treatment efficiency [14-16, 21, 22]. Kidd et al. treated 452 cervical cancer patients with curative intent radiotherapy (135 with PET/CT guided definitive IMRT and 317 non-IMRT). IMRT group showed better OS and cause-specific survival $(p<0.0001)$. The incidences of zgrade 3 bowel or bladder toxicity were $6 \%$ and $17 \%$ for patients treated with IMRT and non-IMRT, respectively $(p=0.0017)$ [21]. Considering the poor tolerance to CCRT, elderly cervical cancer patients may benefit more from new treatment approach like IGBT and IMRT.

Concurrent chemotherapy would significantly increase acute toxicity, which might be untolerated by some elderly cervical cancer patients. However, there is no guideline to recommend who should receive CCRT. Doctors may be confused about that. And this leads to some elderly patients who could tolerate CCRT receive 
less aggressively treatment. Comprehensive geriatric assessment (CGA) is a tool which covers assessment of ability to self-care, mobility and risk of falls, comorbidities, polypharmacy, nutritional status, cognitive function, psychological status, social support, and geriatric syndrome of elderly individuals. A CGA can predict morbidity and mortality in elder patients with cancer [23]. With a CGA, physicians can predict the benefit and risk of treatment and gave personalized treatment to individual patient. It was reported that, elderly cancer patients with prefrail/frail determined by a CGAderived deficit-accumulation index were more likely to have zgrade 3 chemotherapy toxicity [24]. Maybe because of the comparatively small population of elderly cervical cancer patients, the studies on the application of CGA in elderly cervical cancer patients are limited. For elderly local advanced cervical cancer patients, maybe CGA is an approach to look for the patients who could tolerate and benefit from CCRT.

\section{Conclusions}

In our study, elderly cervical cancer patients could tolerate radical RT and CCRT very well and get a good survival. Compared with RT, CCRT could improve the survival of elderly cervical cancer patients with similar nonhematological toxicity. This indicated that CCRT should be considered for elderly cervical cancer patients. This study was a retrospective study. A prospective study is needed to determine the role of CCRT in this population. We also need more studies to look for the patients who could tolerate and benefit from CCRT in the future. CGA may be an approach to look for these patients.

\section{Abbreviations}

3D-CRT: 3-Dimensional conformal radiation therapy; CCRT: Concurrent chemoradiotherapy; CGA: Comprehensive geriatric assessment; Cl: Confidence interval; CR: Completely response; CSS: Cause-specific survival; CTCAE: Common Terminology Criteria for Adverse Events; CTV: Clinical target volume; DFS: Disease-free survival; EBRT: External beam radiation therapy; FIGO: International Federation of Gynecology and Obstetrics; Gl: Gastrointestinal; GTVnd: Gross tumor volume; GU: Genitourinary; HR: Hazard ratio; ICBT: Intracavitary brachytherapy; ICRU: International Commission of Radiation Units; IMRT: Intensity modulated radiation therapy; MRI: Magnetic resonance imaging; NCCN: National Comprehensive Cancer Network; OS: Overall survival; PGTVnd: Planning gross tumor volume; PTV: Planning target volume; PUMCH: Peking Union Medical College Hospital; RT: Radiotherapy; SEER: Surveillance, Epidemiology, and End Results

\section{Acknowledgements}

None.

\section{Funding}

This study is supported by Ministry of Science and Technology of the People's Republic of China (grant number 2016YFC0105207). The funding body played no role in the study design, data collection, data analysis, data interpretation or the writing of the manuscript.

\section{Availability of data and materials}

The datasets used and analyzed during the current study are available from the corresponding author on reasonable request.

\section{Authors' contributions}

WW was responsible for data collection and drafted the manuscript; $X H_{\text {, JS }}$ $X L, J Y, S S, Z L, M Z$ participated in the design of the study. QM, DW, JQ performed statistic analysis and data interpretation; $\mathrm{KH}$ and $\mathrm{FZ}$ designed the study and revised the manuscript. All authors read and approved the manuscript.

\section{Authors' information}

All authors come from department of radiation oncology, Peking Union Medical College Hospital. Chinese Academy of Medical Sciences \& Peking Union Medical College, Beijing, People's Republic of China.

\section{Ethics approval and consent to participate}

The Institutional Review Board (IRB) of Peking Union Medical College Hospital (PUMCH) reviewed the protocol. This is retrospective study. The protocol is rational and scientific. The study accords with principle of ethics. The IRB thus approve the protocol.

The ethical committee process number is S-K214.

This is a retrospective study and written human subject consent was unnecessary.

\section{Consent for publication}

Not applicable.

\section{Competing interests}

The authors declare that they have no competing interests.

\section{Publisher's Note}

Springer Nature remains neutral with regard to jurisdictional claims in published maps and institutional affiliations.

Received: 28 February 2017 Accepted: 24 July 2017

Published online: 01 August 2017

\section{References}

1. Chen W, Zheng R, Baade PD, Zhang S, Zeng H, Bray F, et al. Cancer statistics in China, 2015. CA Cancer J Clin. 2016;66:115-32.

2. Skaznik-Wikiel ME, Sukumvanich P, Austin RM, Zorn KK, Krivak TC, Edwards $\mathrm{RP}$, et al. Heavy cervical cancer burden in elderly women: how can we improve the situation? Acta Cytol. 2012;56:388-93.

3. Sharma C, Deutsch I, Horowitz DP, Hershman DL, Lewin SN, Lu YS, et al. Patterns of care and treatment outcomes for elderly women with cervical cancer. Cancer. 2012:118:3618-26.

4. Nogueira-Rodrigues A, de Melo AC, Garces AH, Paulino E, Alves FV, Vilaca Mdo $\mathrm{N}$, et al. Patterns of care and outcome of elderly women diagnosed with cervical cancer in the developing world. Int J Gynecol Cancer. 2016;26:1246-51.

5. National Comprehensive Cancer Network(NCCN). Clinical Practice Guidelines in Oncology. Cervical Cancer.Version 1. 2017. http://www.nccn.org/ professionals/physician_gls/f_guidelines.asp - cervical. Accessed 25 June 2017.

6. Goodheart M, Jacobson G, Smith BJ, Zhou L. Chemoradiation for invasive cervical cancer in elderly patients: outcomes and morbidity. Int J Gynecol Cancer. 2008;18:95-103.

7. Chakraborty S, Geetha M, Dessai S, Patil VM. How well do elderly patients with cervical cancer tolerate definitive radiochemotherapy using RapidArc? Results from an institutional audit comparing elderly versus younger patients. Ecancermedicalscience. 2014:8:484.

8. Park JH, Kim YS, Ahn SD, Choi EK, Shin SS, Kim YT, et al. Concurrent chemoradiotherapy or radiotherapy alone for locally advanced cervical cancer in elderly women. Tumori. 2010;96:959-65.

9. Wright JD, Gibb RK, Geevarghese S, Powell MA, Herzog TJ, Mutch DG, et al. Cervical carcinoma in the elderly: an analysis of patterns of care and outcome. Cancer. 2005;103:85-91.

10. Ikushima H, Takegawa Y, Osaki K, Furutani S, Yamashita K, Kawanaka T, et al. Radiation therapy for cervical cancer in the elderly. Gynecol Oncol. 2007;107:339-43.

11. Sakurai H, Mitsuhashi N, Takahashi M, Yamakawa M, Akimoto T, Hayakawa K, et al. Radiation therapy for elderly patient with squamous cell carcinoma of the uterine cervix. Gynecol Oncol. 2000;77:116-20. 
12. Wang YM, Wang CJ, Fang FM, Chen HC, Hsu HC, Huang YJ, et al. Differences in the outcomes and complications between elderly and younger uterine cervical cancer patients treated by definitive radiotherapy a propensity score-matched study. Gynecol Oncol. 2017;145:277-83.

13. Chemoradiotherapy for Cervical Cancer Meta-Analysis C. Reducing uncertainties about the effects of chemoradiotherapy for cervical cancer: a systematic review and meta-analysis of individual patient data from 18 randomized trials. J Clin Oncol. 2008;26:5802-12.

14. Hui B, Zhang Y, Shi F, Wang J, Wang T, Wang J, et al. Association between bone marrow dosimetric parameters and acute hematologic toxicity in cervical cancer patients undergoing concurrent chemoradiotherapy: comparison of three-dimensional conformal radiotherapy and intensitymodulated radiation therapy. Int J Gynecol Cancer. 2014;24:1648-52.

15. Chang Y, Yang ZY, Li GL, Li Q, Yang Q, Fan JQ, et al. Correlations between radiation dose in bone marrow and hematological toxicity in patients with cervical cancer: a comparison of 3DCRT, IMRT, and RapidARC. Int I Gynecol Cancer. 2016;26:770-6.

16. Mell LK, Sirak I, Wei L, Tarnawski R, Mahantshetty U, Yashar CM, et al. Bone marrow-sparing intensity modulated radiation therapy with concurrent cisplatin for stage IB-IVA cervical cancer: an international multicenter phase II clinical trial (INTERTECC-2). Int J Radiat Oncol Biol Phys. 2017;97:536-45.

17. Tanderup K, Fokdal LU, Sturdza A, Haie-Meder C, Mazeron R, van Limbergen $E$, et al. Effect of tumor dose, volume and overall treatment time on local control after radiochemotherapy including MRI guided brachytherapy of locally advanced cervical cancer. Radiother Oncol. 2016;120:441-6.

18. Sturdza A, Potter R, Fokdal LU, Haie-Meder C, Tan LT, Mazeron R, et al. Image guided brachytherapy in locally advanced cervical cancer: improved pelvic control and survival in RetroEMBRACE, a multicenter cohort study. Radiother Oncol. 2016;120:428-33.

19. Charra-Brunaud C, Harter V, Delannes M, Haie-Meder C, Quetin P, Kerr C, et al. Impact of 3D image-based PDR brachytherapy on outcome of patients treated for cervix carcinoma in France: results of the French STIC prospective study. Radiother Oncol. 2012;103:305-13.

20. Potter R, Georg P, Dimopoulos JC, Grimm M, Berger D, Nesvacil N, et al. Clinical outcome of protocol based image (MRI) guided adaptive brachytherapy combined with 3D conformal radiotherapy with or without chemotherapy in patients with locally advanced cervical cancer. Radiother Oncol. 2011;100:116-23.

21. Kidd EA, Siegel BA, Dehdashti F, Rader JS, Mutic S, Mutch DG, et al. Clinical outcomes of definitive intensity-modulated radiation therapy with fluorodeoxyglucose-positron emission tomography simulation in patients with locally advanced cervical cancer. Int J Radiat Oncol Biol Phys. 2010;77:1085-91.

22. Gandhi AK, Sharma DN, Rath GK, Julka PK, Subramani V, Sharma S, et al. Early clinical outcomes and toxicity of intensity modulated versus conventional pelvic radiation therapy for locally advanced cervix carcinoma: a prospective randomized study. Int J Radiat Oncol Biol Phys. 2013;87:542-8.

23. Extermann M, Hurria A. Comprehensive geriatric assessment for older patients with cancer. J Clin Oncol. 2007;25:1824-31.

24. Cohen HJ, Smith D, Sun CL, Tew W, Mohile SG, Owusu C, et al. Frailty as determined by a comprehensive geriatric assessment-derived deficitaccumulation index in older patients with cancer who receive chemotherapy. Cancer. 2016;122:3865-72.

\section{Submit your next manuscript to BioMed Central and we will help you at every step:}

- We accept pre-submission inquiries

- Our selector tool helps you to find the most relevant journal

- We provide round the clock customer support

- Convenient online submission

- Thorough peer review

- Inclusion in PubMed and all major indexing services

- Maximum visibility for your research

Submit your manuscript at www.biomedcentral.com/submit
Biomed Central 